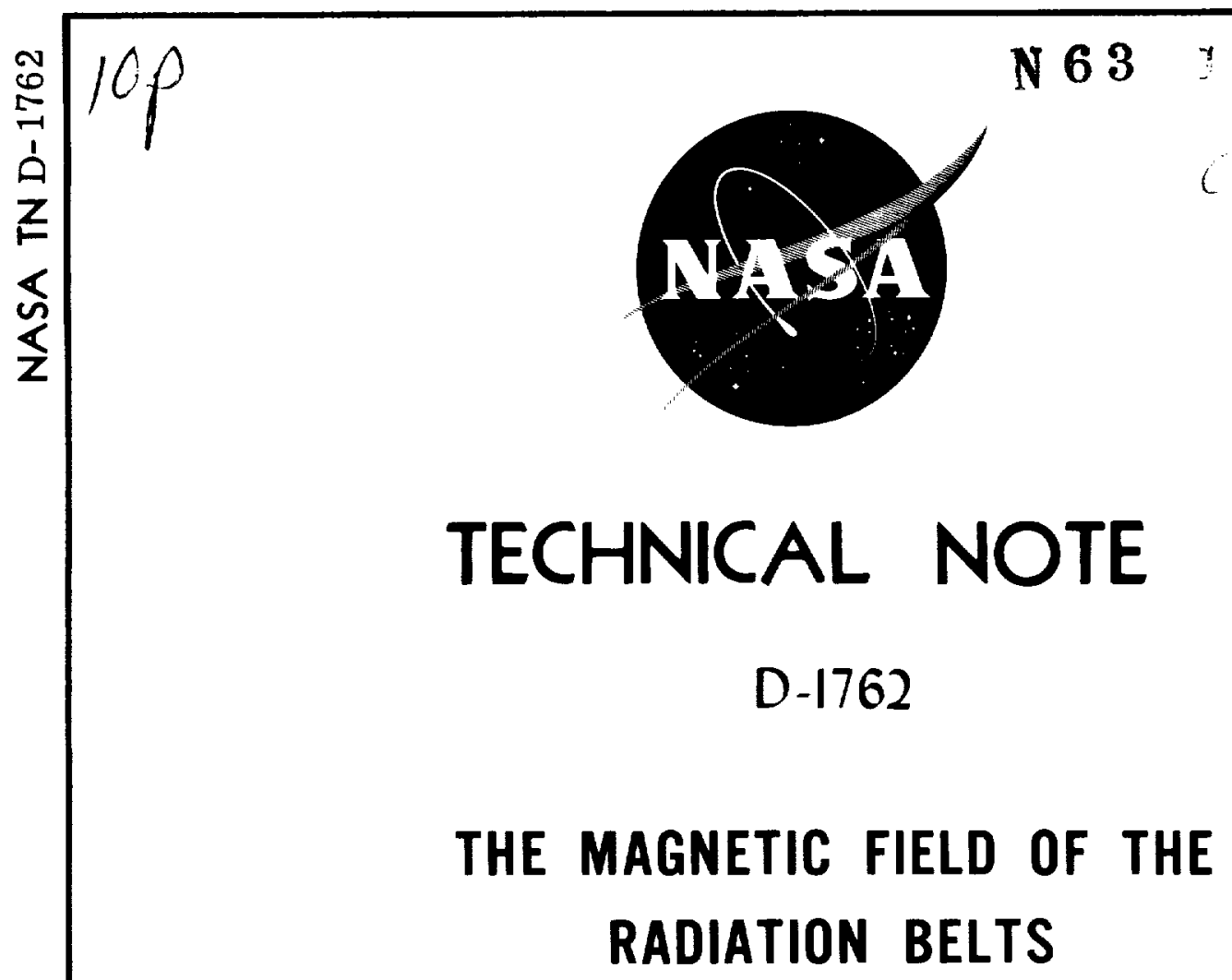

\author{
Syun-Ichi Akasofu \\ University of Alaska \\ College, Alaska \\ Joseph C. Cain
}

Goddard Space Flight Center

Greenbelt, Maryland

NATIONAL AERONAUTICS AND SPACE ADMINISTRATION 
Codi.'

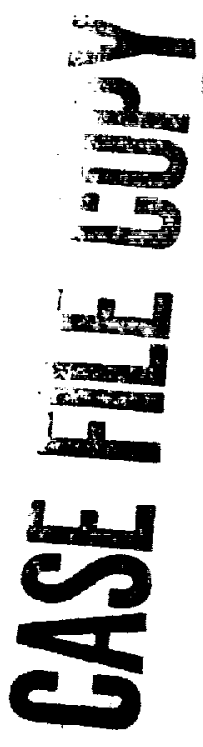




\title{
THE MAGNETIC FIELD OF THE RADIATION BELTS
}

\author{
by \\ Syun-Ichi Akasofu \\ University of Alaska \\ Joseph C. Cain \\ Goddard Space Flight Center
}

SUMMARY

The magnetic fields produced by various types of belts of trapped particles are presented in both tabular and graphical forms. 



\title{
THE MAGNETIC FIELD OF THE RADIATION BELTS*
}

\author{
by \\ Syun-Ichi Akasofu \\ University of Alaska \\ Joseph C. Cain \\ Goddard Space Flight Center
}

In this note we shall present in graphical form the magnetic fields produced by various types of belts of trapped particles. The chosen values of the parameters determining the field cover a wide range. It is hoped that, by interpolating the diagrams shown, the distortion of the earth's field can be approximately estimated for actually observed belts without a large amount of computation. References 1-5 discuss the method of computation.

Herein we shall state briefly the formulas used. The number density distribution along an equatorial radius $r: N=f_{1}(r)$ is given by:

$$
\begin{aligned}
& N=N_{0} e^{-z_{1}^{2} z^{2}}(z<0 \text { for the inner part of the belt }) ; \\
& N=N_{0} e^{-z_{2}^{2} z^{2}}(z>0 \text { for the outer part of the belt }),
\end{aligned}
$$

where $z=\left(r-r_{0}\right) / a$, a is the radius of the earth, and $r_{0}$ denotes the distance at which $N$ attains its maximum value $\mathrm{N}_{0}$. Thus we may write

$$
N=f_{1}\left(r_{0}, z, g_{1}, g_{2}\right) .
$$

The pitch-angle distribution, $\mathbf{P}=\mathbf{f}_{2}(\theta, a)$, is

$$
P=A(\alpha): \sin ^{\alpha+1} \theta \text {, }
$$

where $\theta$ denotes the pitch angle, $a$ is a constant, and $\mathrm{A}(a)$ is a normalization factor.

In the energy spectrum $N=f_{3}(E)$, we shall consider only particles with a particular energy $E$ (or speed $v$ ). The functions $f_{1}, f_{2}$, and $f_{3}$ determine the electric current intensity at any point in a dipole field. The ring current field $\Delta F$ is then calculated by a method shown in Reference 2. 


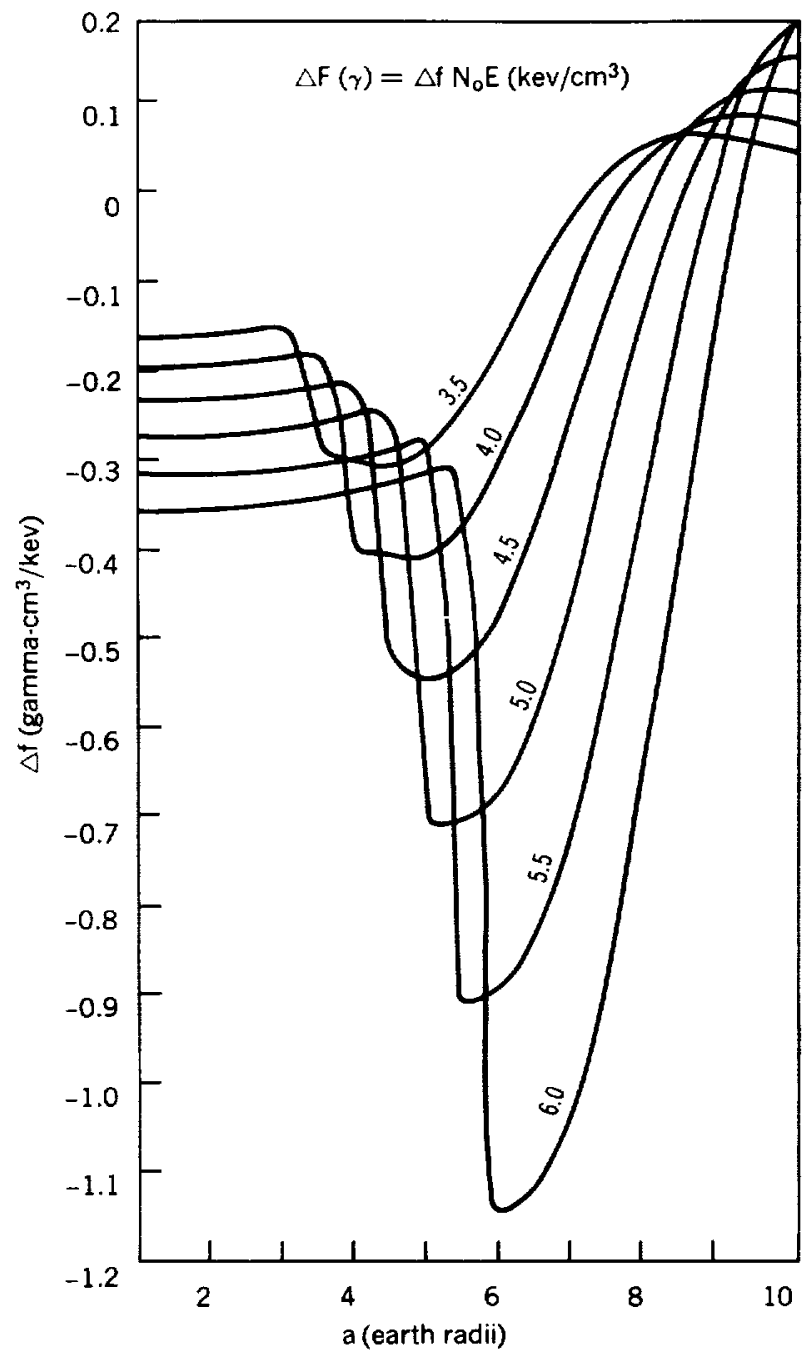

Figure $1(a)$ - Ring current field $\Delta f$ for several values of $r_{0}$.

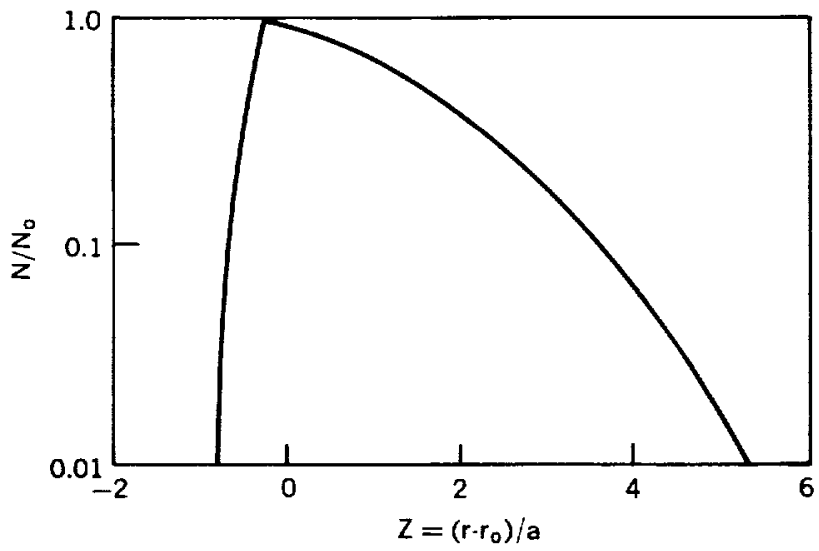

Figure 1(b)-Normalized electron density distribution.
The ring current field $\Delta F$ is given by:

$$
\Delta F=f\left(r, r_{0}, g_{1}, g_{2}, a, N_{0}, E\right) .
$$

In the first approximation, $\Delta F$ is proportional to both $\mathrm{N}_{0}$ and $\mathrm{E}$. Therefore, Equation 1 may be rew ritten

$$
\Delta F=\Delta \mathrm{F} N_{0} E \text {. }
$$

where

$$
\Delta f=f^{\prime}\left(r, r_{0}, g_{1}, g_{2}, a\right) .
$$

We will show $\Delta f$, as a function of equatorial radial distance $r$, for various sets of $r_{0}, g_{1}, g_{2}$, and $a$. The vector $\Delta F$ is perpendicular to the equatorial plane and its value of $\Delta F$ is given in gammas, if the units of $\mathrm{N}_{0}$ and $\mathrm{E}$ are chosen as $\mathrm{cm}^{-3}$ and kev, respectively.

In Figure 1(a) $\Delta f$ is shown for different values of $r_{0}$ (Appendix $A$ ). The other parameters $\mathrm{g}_{1}, \mathrm{~g}_{2}$, and a are chosen in such a way that they are suitable for the quiet-time proton belt (Reference 3 ):

$$
\begin{aligned}
& \mathrm{g}_{1}=2.990\left(\mathrm{~N} / \mathrm{N}_{0}=1 / 10 \text { at } \mathrm{z}=-0.51\right) \\
& \mathrm{g}_{2}=0.419\left(\mathrm{~N} / \mathrm{N}_{0}=1 / 10 \text { at } \mathrm{z}=+3.62\right), \\
& a=2.0 .
\end{aligned}
$$

The graph of $\mathrm{N} / \mathrm{N}_{0}$ is given in Figure $1(\mathrm{~b})$.

Figure 2(a) presents $\Delta f$ for different values of $\mathrm{g}_{2}$, namely:

$$
\begin{aligned}
& \mathrm{g}_{2}=2.146\left(\mathrm{~N} / \mathrm{N}_{0}=1 / 100 \text { at } \mathrm{z}=+1\right) . \\
& \mathrm{g}_{2}=1.517\left(\mathrm{~N} / \mathrm{N}_{0}=1 / 10 \text { at } \mathrm{z}=+1\right) .
\end{aligned}
$$


$\mathrm{g}_{2}=0.759\left(\mathrm{~N} / \mathrm{N}_{0}=1 / 10\right.$ at $\left.z=+2\right)$,
$\mathrm{g}_{2}=0.379\left(\mathrm{~N} / \mathrm{N}_{0}=1 / 10\right.$ at $\left.z=+4\right)$.

The graph of $\mathrm{N} / \mathrm{N}_{0}$ for these four cases is shown in Figure $2(\mathrm{~b})$. The other parameters are chosen as follows:

$$
\begin{aligned}
& \mathbf{r}_{0}=3.2 \mathrm{a} \\
& \mathrm{g}_{1}=2.146\left(\mathrm{~N} / \mathrm{N}_{0}=1 / 100 \text { at } z=-1\right), \\
& a=2.0
\end{aligned}
$$

In Figure $3(\mathrm{a}) \Delta \mathrm{f}$ is given for different values of $a$; the graph of $P / A$ for various values of $a$ is given in Figure 3(b). The other parameters are chosen as follows:

$r_{0}=3.2 \mathrm{a}$

$\mathrm{g}_{1}=2.628\left(\mathrm{~N} / \mathrm{N}_{0}=1 / 1000\right.$ at $\left.\mathrm{z}=-1\right)$,

$\mathrm{g}_{2}=0379\left(\mathrm{~N} / \mathrm{N}_{0}=1 / 10\right.$ at $\left.\mathrm{z}=+4\right)$.

The tabulated data from which Figures 1(a), 2(a), and 3(a) are plotted are presented in Appendix A.

\section{REFERENCES}

1. Akasofu, S.-I., and Chapman, S., "The Ring Current, Geomagnetic Disturbance and the Van Allen Radiation Belts,"J.Geophys. Res. 66(5):1321-1350, May 1961.

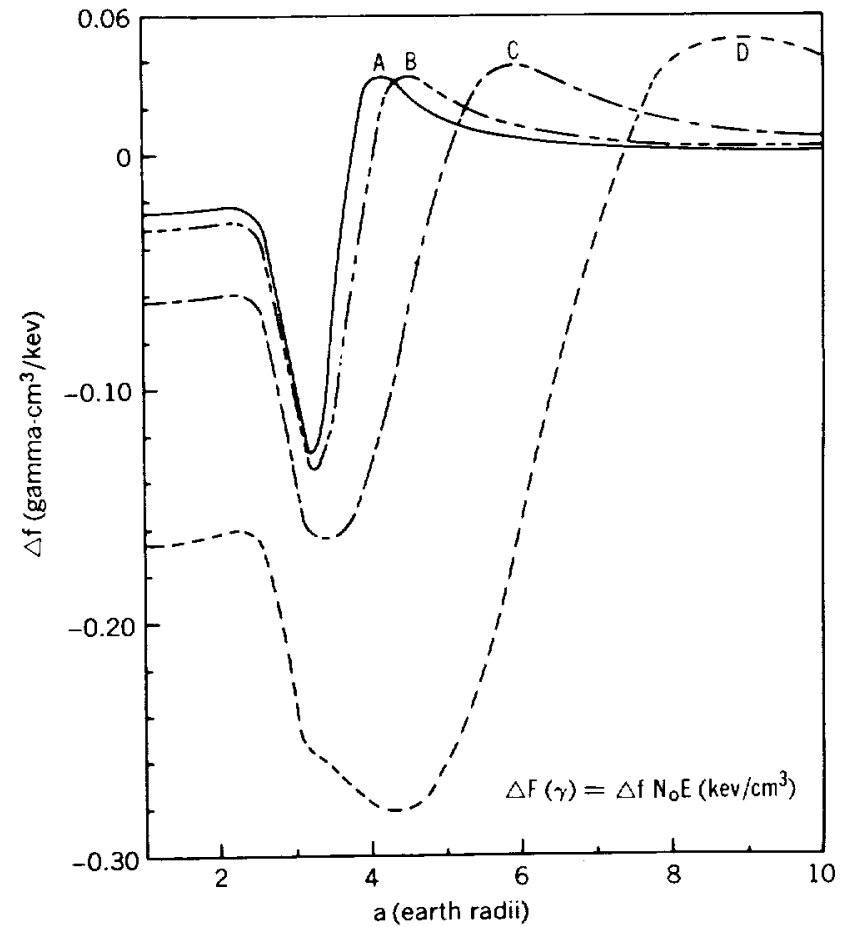

Figure 2(a)-Ring current field $\Delta f$ for several values of outer slope. The values of $g_{2}$ for curves $A, B, C$, and $D$ are $2.146,1.517,0.759$ and 0.379 respectively.

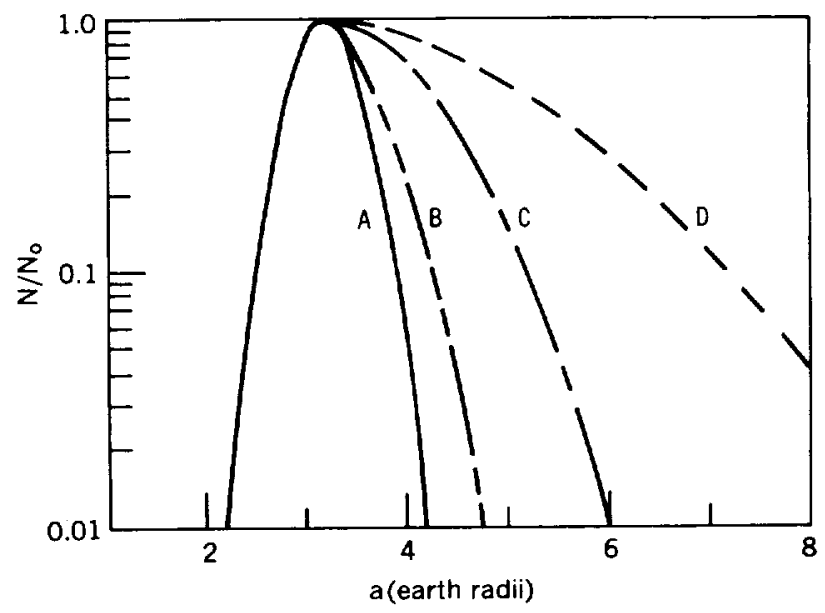

Figure 2(b) - The normalized electron density distribution. The curves $A, B, C$, and $D$ are defined in Figure $4 a$.

2. Akasofu, S.-I., Cain, J. C., and Chapman, S., "The Magnetic Field of a Radiation Belt, Numerically Computed," NASA Technical Note D-1447, November 1962; also J. Geophys. Res. 66(12):4013-4020, December 1961.

3. Akasofu, S.-I., Cain, J. C., and Chapman, S., "The Magnetic Field of the Quiet-Time Proton Belt," NASA Technical Note D-1674, 1963; also J. Geophys. Res. 67(7):2645-2647, July 1962. 


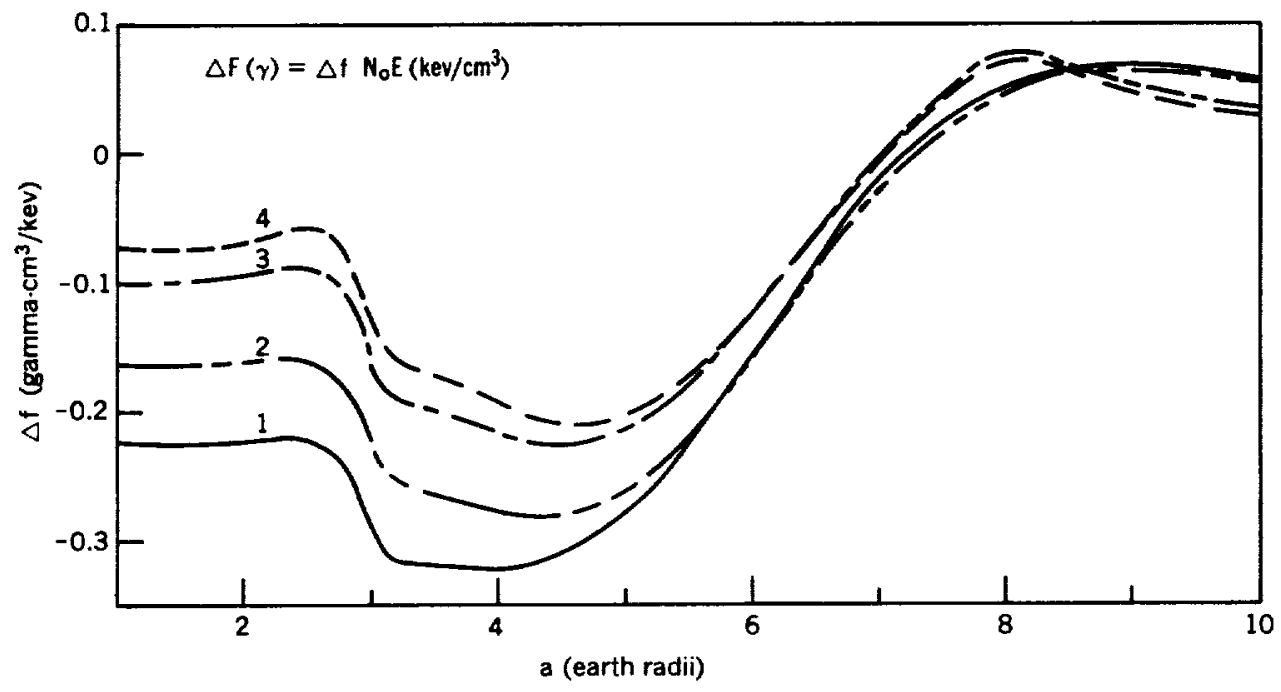

Figure 3(a)-Ring current field $\Delta f$ for several $a$.

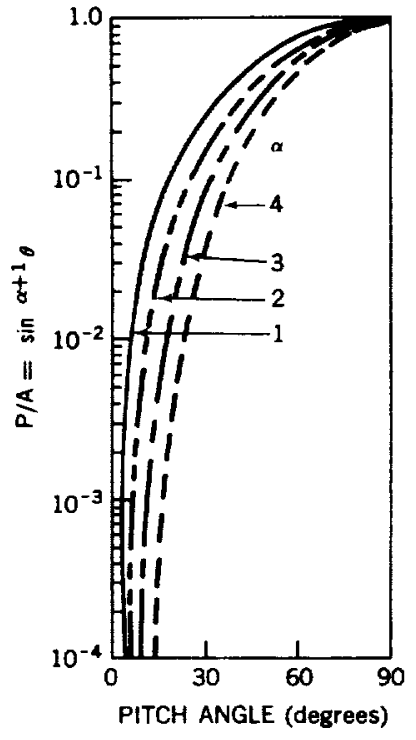

Figure $3(b)-N o r m a l i z e d$ pitch angle distribution for various a.

4. Beard, D. B., "Self-Consistent Calculation of the Ring Current," J. Geophys. Res. 67(9):3615-3616, August 1962.

5. Akasofu, S.-I., "On a Self-Consistent Calculation of the Ring Current Field," J. Geophys. Res. 67(9): 3617-3618, August 1962. 
Appendix A

The Ring Current Field $\Delta f$

as a Function of $r, g_{2}$, and $a$ 
The Value of $\Delta f$ as a Function of $r_{0}$.

\begin{tabular}{|c|c|c|c|c|c|c|c|c|c|c|}
\hline \multirow{2}{*}{$\begin{array}{c}r \\
\text { (earth } \\
\text { radii) }\end{array}$} & \multicolumn{10}{|c|}{ Ring current field (gamma) } \\
\hline & $r_{0}=1.5 a$ & $r_{0}=2.00$ & $1_{0}=2.5 a$ & $r_{0}=3.0 \mathrm{a}$ & $r_{0}=3.5 a$ & $r_{0}=4.0 a$ & $r_{0}=4.5 a$ & $r_{0}=5.0 \mathrm{a}$ & $r_{0}=5.5 a$ & $r_{0}=6.0 a$ \\
\hline 1.0 & -0.0578 & -0.0779 & -0.1020 & -0.1296 & -0.1604 & 2 & -0.2334 & -0.2744 & -0.3169 & -0.3576 \\
\hline 1.1 & -0.0577 & -0.0778 & -0.1019 & -0.1295 & -0.1602 & -0.1950 & -0.2332 & -0.2743 & -0.3167 & -0.3574 \\
\hline 1.2 & -0.0576 & -0.0777 & -0.1017 & -0.1294 & -0.1600 & -0.1948 & -0.2330 & -0.2741 & -0.3166 & -0.3572 \\
\hline 1.3 & -0.0574 & -0.0775 & -0.1016 & -0.1292 & -0.1597 & -0.1946 & -0.2328 & -0.2738 & -0.3163 & -0.3570 \\
\hline 1.4 & -0.0572 & -0.0773 & -0.1014 & -0.1291 & -0.1594 & -0.1943 & -0.2328 & -0.2736 & -0.3161 & -0.3568 \\
\hline 1.5 & -0.0576 & -0.0771 & -0.1012 & -0.1289 & -0.1591 & -0.1940 & -0.2323 & -0.2733 & -0.3158 & -0.3565 \\
\hline 1.6 & -0.0591 & -0.0769 & -0.1010 & -0.1287 & -0.1587 & -0.1937 & -0.2319 & -0.2730 & -0.3156 & -0.3562 \\
\hline 1.7 & -0.0614 & -0.0766 & -0.1007 & -0.1284 & -0.1583 & -0.1933 & -0.2316 & -0.2727 & -0.3152 & -0.3559 \\
\hline 1.8 & -0.0640 & -0.0766 & -0.1004 & -0.1281 & -0.1578 & -0.1928 & -0.2312 & -0.2723 & -0.3149 & -0.3556 \\
\hline 1.9 & -0.0654 & -0.0773 & -0.1000 & -0.1278 & -0.1572 & -0.1924 & -0.2308 & -0.2719 & -0.3145 & -0.3552 \\
\hline 2.0 & -0.0662 & -0.0794 & -0.0996 & -0.1274 & -0.1566 & -0.1918 & -0.2303 & -0.2715 & -0.3141 & -0.3548 \\
\hline 2.1 & -0.0671 & -0.0836 & -0.0991 & -0.1270 & -0.1559 & -0.1912 & -0.2297 & -0.2710 & -0.3136 & -0.3543 \\
\hline 2.2 & -0.0683 & -0.0898 & -0.0988 & -0.1265 & -0.1552 & & -0.2292 & -0.2705 & -0.3131 & -0.3539 \\
\hline 2.3 & -0.0696 & -0.0961 & -0.0991 & -0.1259 & -0.1543 & -0.1898 & -0.2285 & -0.2699 & -0.3126 & -0.3534 \\
\hline 2.4 & -0.0709 & -0.0992 & -0.1010 & -0.1253 & -0.1534 & -0.1890 & -0.2278 & -0.2693 & -0.3120 & -0.3528 \\
\hline 2.5 & -0.0723 & -0.1002 & $-0.106 \mid$ & -0.1246 & -0.1523 & -0.1881 & -0.2270 & -0.2686 & -0.3114 & -0.3522 \\
\hline 2.6 & -0.0737 & -0.1017 & -0.1156 & -0.1240 & -0.1512 & -0.1872 & -0.2262 & -0.2678 & -0.3107 & -0.3515 \\
\hline 2.7 & -0.0751 & -0.1 & -0.1 & -0.1237 & -0.1502 & -0.1861 & -0.2253 & -0.2870 & -0.3099 & -0.3508 \\
\hline 2.8 & -0.0764 & -0.1051 & -0.1415 & -0.1247 & -0.1498 & -0.1849 & -0.2243 & -0.2661 & $-0.309 \mid$ & -0.3501 \\
\hline 2.9 & -0.0776 & -0.1070 & -0.1472 & -0.1288 & -0.1517 & -0.1836 & -0.2231 & -0.2651 & -0.3083 & -0.3493 \\
\hline 3.0 & -0.0786 & -0.1 & -0.1 & -0. & -0.1 & -0. & -0.2219 & -0.2641 & -0.3073 & -0.3484 \\
\hline 3.1 & -0.0794 & -0. & -0.1502 & -0.1569 & -0.1761 & -0.1807 & -0.2206 & -0.2629 & -0.3063 & -0.3474 \\
\hline 3.2 & -0.0800 & -0.1123 & -0.1522 & -0.1810 & -0.2061 & -0.1795 & -0.2192 & -0.2617 & -0.3052 & -0.3464 \\
\hline $\begin{array}{l}.2 \\
3.3\end{array}$ & -0.0804 & -0.1138 & -0.1544 & -0.2034 & -0.2452 & -0.1794 & -0.2177 & -0.2604 & -0.3040 & -0.3454 \\
\hline 3.4 & -0.0804 & -0.1150 & -0.1567 & -0.2130 & -0.2807 & -0.1831 & -0.2160 & -0.2589 & -0.3028 & -0.3442 \\
\hline 3.5 & -0.0 & 0 & -0.1 & -0 & -0.2 & & -0.2142 & -0.2874 & -0.3014 & -0.3430 \\
\hline 3.6 & -0.0794 & -0.1165 & -0.1609 & -0.2160 & -0.2955 & -0.2230 & -0.2124 & -0.2557 & -0.2999 & -0.3416 \\
\hline 3.7 & -0.0784 & -0.1167 & -0.1626 & -0.2183 & -0.2969 & -0.2 & -0.2110 & -0.2539 & -0.2983 & -0.3402 \\
\hline 3.8 & $\begin{array}{l}-0.0704 \\
-0.0771\end{array}$ & -0.1164 & -0.1639 & -0.2207 & -0.2989 & -0.3304 & -0.2114 & -0.2520 & -0.2967 & -0.3387 \\
\hline 3.9 & -0.0753 & -0.1156 & -0.1648 & -0.2231 & -0.3011 & -0.3842 & -0.2176 & -0.2499 & -0.2948 & -0.3371 \\
\hline 4.0 & -0.0 & 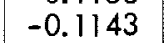 & -0 & -0 & & -0 & 10 & -0.2476 & -0.2929 & -0.3353 \\
\hline 4.1 & -0.0707 & -0.1126 & -0.1651 & -0.2270 & -0.3049 & -0.4056 & -0.2784 & -0.2455 & -0.2908 & -0.3335 \\
\hline 4.2 & -0.0679 & -0.1103 & -0.1643 & -0.2284 & -0.3062 & -0.40 & -0.3480 & -0.2440 & -0.2886 & -0.3315 \\
\hline 4.3 & -0.0648 & -0.1075 & -0.1628 & -0.2292 & -0.3067 & -0.4078 & -0.4361 & -0.2453 & -0.2862 & -0.3294 \\
\hline 4.4 & -0.0614 & -0.1041 & -0.1607 & -0.2293 & -0.3065 & -0.4094 & -0.5135 & -0.2550 & -0.2837 & -0.3272 \\
\hline 4.5 & -0.0578 & -0.10 & -0.1579 & -0.2286 & -0.3053 & -0.410 & -0.5439 & -0.2832 & -0.2810 & -0.3248 \\
\hline 4.6 & -0.0540 & -0.0961 & -0.1543 & -0.2271 & -0.3031 & -0.4115 & -0.5437 & -0.3431 & -0.2785 & -0.3223 \\
\hline 4.7 & -0.0500 & -0.0915 & -0.1501 & -0.2247 & -0.2997 & -0.4115 & -0.5435 & -0.4414 & -0.2770 & -0.3196 \\
\hline 4.8 & -0.0458 & -0.0864 & -0.1452 & -0.2215 & -0.2952 & -0.4105 & -0.5439 & -0.5643 & -0.2796 & -0.3168 \\
\hline 4.9 & -0.0416 & -0.081 & -0.1397 & -0.2173 & -0.2896 & -0.4084 & -0.5443 & -0.6710 & -0.2939 & -0.3137 \\
\hline 5.0 & -0.0373 & -0.0754 & -0.1335 & -0.2121 & -0.2827 & -0.4050 & -0.5442 & -0.7131 & -0.3337 & -0.3106 \\
\hline 5.5 & -0.0167 & -0.0453 & -0.0958 & -0.1733 & -0.2315 & -0.3661 & -0.5265 & -0.7064 & -0.9150 & -0.3853 \\
\hline 6.0 & -0.0002 & -0.0168 & -0.0532 & -0.1190 & -0.1601 & -0.2916 & -0.4658 & -0.6720 & -0.8990 & -1.1490 \\
\hline 6.5 & 03 & +0.0050 & -0.0150 & -0.0610 & -0.0838 & -0.1944 & -0.3619 & -0.5831 & -0.8426 & -1.1220 \\
\hline 7.0 & +0.0153 & +0.0181 & +0.0130 & -0.0 & -0.0175 & -0.0946 & -0.2330 & -0.4427 & -0.7182 & -1.0370 \\
\hline 7.5 & +0.0162 & +0.0237 & +0.0290 & +0.0243 & +0.0296 & -0.0106 & -0.1050 & -0.2757 & -0.5335 & -0.8692 \\
\hline 8.0 & +0.0149 & +0.0242 & +0.0352 & +0.0436 & +0.0560 & +0.0475 & -0.0002 & -0.1144 & -0.3211 & -0.6314 \\
\hline 8.5 & +0.0128 & +0.0219 & +0.0348 & +0.0502 & +0.0654 & +0.0787 & +0.0705 & +0.0148 & -0.1209 & -0.3656 \\
\hline 9.0 & +0.0106 & +0.0187 & +0.0312 & +0.0487 & +0.0641 & +0.0889 & +0.1073 & +0.1000 & +0.0365 & -0.1200 \\
\hline 9.5 & +0.0087 & +0.0155 & +0.0266 & +0.0434 & +0.0573 & +0.0859 & +0.1182 & +0.1430 & +0.1384 & +0.0700 \\
\hline 10.0 & +0.0071 & +0.0128 & +0.0222 & +0.0370 & +0.0490 & +0.0766 & +0.1131 & +0.1545 & +0.1885 & +0.1910 \\
\hline
\end{tabular}


Table A2

The Values of $\Delta f$ as a function of $g_{2}$.

\begin{tabular}{|c|c|c|c|c|c|}
\hline \multirow{2}{*}{$($ earth radii) } & \multicolumn{5}{|c|}{ Ring current field (gamma) } \\
\hline & $g_{2}=2.146$ & $g_{2}=1.517$ & $\mathrm{~g}_{2}=0.759$ & $g_{2}=0.509$ & $g_{2}=0.379$ \\
\hline $\begin{array}{l}1.0 \\
1.1 \\
1.2 \\
1.3 \\
1.4 \\
1.5 \\
1.6 \\
1.7 \\
1.8 \\
1.9 \\
2.0 \\
2.1 \\
2.2 \\
2.3 \\
2.4 \\
2.5 \\
2.6 \\
2.7 \\
2.8 \\
2.9 \\
3.0 \\
3.1 \\
3.2 \\
3.3 \\
3.4 \\
3.5 \\
3.6 \\
3.7 \\
3.8 \\
3.9 \\
4.0 \\
4.1 \\
4.2 \\
4.3 \\
4.4 \\
4.5 \\
4.6 \\
4.7 \\
4.8 \\
4.9 \\
5.0 \\
5.5 \\
6.0 \\
6.5 \\
7.0 \\
7.5 \\
8.0 \\
8.5 \\
9.0 \\
9.5 \\
10.0\end{array}$ & $\begin{array}{l}-0.0245 \\
-0.0245 \\
-0.0243 \\
-0.0242 \\
-0.0241 \\
-0.0239 \\
-0.0237 \\
-0.0235 \\
-0.0233 \\
-0.0230 \\
-0.0227 \\
-0.0224 \\
-0.0223 \\
-0.0226 \\
-0.0240 \\
-0.0273 \\
-0.0339 \\
-0.0449 \\
-0.0610 \\
-0.0812 \\
-0.1024 \\
-0.1199 \\
-0.1282 \\
-0.1237 \\
-0.1061 \\
-0.0786 \\
-0.0469 \\
-0.0171 \\
+0.0066 \\
+0.0225 \\
+0.0310 \\
+0.0339 \\
+0.0334 \\
+0.0312 \\
+0.0283 \\
+0.00013 \\
+0.0255 \\
+0.0228 \\
+0.0205 \\
+0.0185 \\
+0.0168 \\
+0.0152 \\
+0.0100 \\
+0.0070 \\
+0.0051 \\
+0.0039 \\
+0.0030 \\
+0024 \\
+0.0016 \\
+0.011\end{array}$ & $\begin{array}{l}-0.0320 \\
-0.0319 \\
-0.0318 \\
-0.0317 \\
-0.0315 \\
-0.0313 \\
-0.0311 \\
-0.0309 \\
-0.0306 \\
-0.0302 \\
-0.0299 \\
-0.0296 \\
-0.0294 \\
-0.0297 \\
-0.0310 \\
-0.0342 \\
-0.0407 \\
-0.0516 \\
-0.0676 \\
-0.0876 \\
-0.1087 \\
-0.1259 \\
-0.1341 \\
-0.1335 \\
-0.1261 \\
-0.1123 \\
-0.0931 \\
-0.0706 \\
-0.0470 \\
-0.0245 \\
-0.0050 \\
+0.0106 \\
+0.0218 \\
+0.0290 \\
+0.0328 \\
+0.0341 \\
+0.0335 \\
+0.0319 \\
+0.0017 \\
+0.0298 \\
+0.0274 \\
+0.0251 \\
+0.0162 \\
+0.0111 \\
+0.0080 \\
+0.0060 \\
+0.0047 \\
+0.0030 \\
+0025 \\
+0.0017\end{array}$ & $\begin{array}{l}-0.0638 \\
-0.0637 \\
-0.0635 \\
-0.0633 \\
-0.0631 \\
-0.0628 \\
-0.0625 \\
-0.0621 \\
-0.0617 \\
-0.0613 \\
-0.0607 \\
-0.0603 \\
-0.0599 \\
-0.0600 \\
-0.0610 \\
-0.0640 \\
-0.0702 \\
-0.0808 \\
-0.0964 \\
-0.1160 \\
-0.1366 \\
-0.1534 \\
-0.1611 \\
-0.1630 \\
-0.1640 \\
-0.1637 \\
-0.1618 \\
-0.1580 \\
-0.1523 \\
-0.1447 \\
-0.1352 \\
-0.1240 \\
-0.1114 \\
-0.0978 \\
-0.0835 \\
-0.0688 \\
-0.0543 \\
-0.0402 \\
-0.0269 \\
-0.0145 \\
-0.0034 \\
+0.0315 \\
+0.0379 \\
+0.0319 \\
+0.0242 \\
+0.0182 \\
+0.0140 \\
+0.0110 \\
+0089 \\
+0.0061\end{array}$ & $\begin{array}{l}-0.1075 \\
-0.1073 \\
-0.1071 \\
-0.1068 \\
-0.1065 \\
-0.1062 \\
-0.1058 \\
-0.1053 \\
-0.1048 \\
-0.1042 \\
-0.1036 \\
-0.1029 \\
-0.1024 \\
-0.1023 \\
-0.1032 \\
-0.1059 \\
-0.1119 \\
-0.1222 \\
-0.1375 \\
-0.1568 \\
-0.1771 \\
-0.1935 \\
-0.2008 \\
-0.2028 \\
-0.2051 \\
-0.2073 \\
-0.2093 \\
-0.2107 \\
-0.2114 \\
-0.2112 \\
-0.2100 \\
-0.2077 \\
-0.2042 \\
-0.1995 \\
-0.1935 \\
-0.1864 \\
-0.1781 \\
-0.1688 \\
-0.1585 \\
-0.1474 \\
-0.1356 \\
-0.0719 \\
-0.0143 \\
+0.0247 \\
+0.0432 \\
+0.0466 \\
+0.0421 \\
+0.0351 \\
+0.0285 \\
+0.0230 \\
+0.0188\end{array}$ & $\begin{array}{l}-0.1663 \\
-0.1661 \\
-0.1658 \\
-0.1655 \\
-0.1651 \\
-0.1647 \\
-0.1642 \\
-0.1636 \\
-0.1630 \\
-0.1623 \\
-0.1616 \\
-0.1608 \\
-0.1601 \\
-0.1599 \\
-0.1606 \\
-0.1631 \\
-0.1688 \\
-0.1789 \\
-0.1940 \\
-0.2131 \\
-0.2331 \\
-0.2491 \\
-0.2560 \\
-0.2579 \\
-0.2604 \\
-0.2634 \\
-0.2665 \\
-0.2697 \\
-0.2727 \\
-0.2755 \\
-0.2779 \\
-0.2797 \\
-0.2810 \\
-0.2815 \\
-0.2813 \\
-0.2802 \\
-0.2783 \\
-0.2754 \\
-0.2716 \\
-0.2668 \\
-0.2610 \\
-0.2186 \\
-0.1592 \\
-0.0940 \\
-0.0343 \\
+0.0118 \\
+0.0415 \\
+0.0561 \\
+0.0596 \\
+0.0565 \\
+0.0502\end{array}$ \\
\hline
\end{tabular}


Table A3

The Values of $\Delta f$ as a Function of $a$.

\begin{tabular}{|c|c|c|c|c|}
\hline \multirow{2}{*}{ (earth radii) } & \multicolumn{4}{|c|}{ Ring current field (gamma) } \\
\hline & $a=1.0$ & $a=2.0$ & $a=3.0$ & $a=4.0$ \\
\hline $\begin{array}{l}1.0 \\
1.1 \\
1.2 \\
1.3 \\
1.4 \\
1.5 \\
1.6 \\
1.7 \\
1.8 \\
1.9 \\
2.0 \\
2.1 \\
2.2 \\
2.3 \\
2.4 \\
2.5 \\
2.6 \\
2.7 \\
2.8 \\
2.9 \\
3.0 \\
3.1 \\
3.2 \\
3.3 \\
3.4 \\
3.5 \\
3.6 \\
3.7 \\
3.8 \\
3.9 \\
4.0 \\
4.1 \\
4.2 \\
4.3 \\
4.4 \\
4.5 \\
4.6 \\
4.7 \\
4.8 \\
4.9 \\
5.0 \\
5.5 \\
6.0 \\
6.5 \\
7.0 \\
7.5 \\
8.0 \\
8.5 \\
9.0 \\
9.5 \\
10.0\end{array}$ & $\begin{array}{l}-0.2240 \\
-0.2239 \\
-0.2239 \\
-0.2239 \\
-0.2238 \\
-0.2237 \\
-0.2237 \\
-0.2235 \\
-0.2234 \\
-0.2232 \\
-0.2230 \\
-0.0228 \\
-0.0226 \\
-0.2224 \\
-0.2226 \\
-0.2238 \\
-0.2273 \\
-0.2350 \\
-0.2489 \\
-0.2692 \\
-0.2929 \\
-0.3126 \\
-0.3197 \\
-0.3193 \\
-0.3196 \\
-0.3202 \\
-0.3210 \\
-0.3217 \\
-0.3223 \\
-0.3225 \\
-0.3222 \\
-0.3214 \\
-0.3200 \\
-0.3178 \\
-0.3149 \\
-0.3111 \\
-0.3064 \\
-0.3009 \\
-0.2944 \\
-0.2871 \\
-0.2788 \\
-0.2254 \\
-0.1581 \\
-0.0881 \\
-0.0263 \\
+0.0202 \\
+0.0493 \\
+0.0630 \\
+0.06515 \\
+0545\end{array}$ & $\begin{array}{l}-0.1649 \\
-0.1647 \\
-0.1644 \\
-0.1641 \\
-0.1638 \\
-0.1634 \\
-0.1629 \\
-0.1624 \\
-0.1618 \\
-0.1611 \\
-0.1604 \\
-0.1596 \\
-0.1587 \\
-0.1578 \\
-0.1572 \\
-0.1576 \\
-0.1605 \\
-0.1679 \\
-0.1821 \\
-0.2036 \\
-0.2292 \\
-0.2511 \\
-0.2599 \\
-0.2611 \\
-0.2631 \\
-0.2656 \\
-0.2685 \\
-0.2714 \\
-0.2742 \\
-0.2768 \\
-0.2790 \\
-0.2807 \\
-0.2819 \\
-0.2823 \\
-0.2820 \\
-0.2809 \\
-0.2789 \\
-0.2759 \\
-0.2721 \\
-0.2672 \\
-0.2614 \\
-0.2189 \\
-0.1594 \\
-0.0941 \\
-0.0344 \\
+0.0117 \\
+0.0414 \\
+0.0560 \\
+0.0596 \\
+0.0564 \\
+0.0501\end{array}$ & $\begin{array}{l}-0.1017 \\
-0.1013 \\
-0.1008 \\
-0.1002 \\
-0.0996 \\
-0.0989 \\
-0.0981 \\
-0.0972 \\
-0.0962 \\
-0.0950 \\
-0.0938 \\
-0.0923 \\
-0.0908 \\
-0.0891 \\
-0.0877 \\
-0.0873 \\
-0.0894 \\
-0.0962 \\
-0.1102 \\
-0.1319 \\
-0.1582 \\
-0.1811 \\
-0.1908 \\
-0.1926 \\
-0.1954 \\
-0.1988 \\
-0.2027 \\
-0.2067 \\
-0.2107 \\
-0.2146 \\
-0.2182 \\
-0.2213 \\
-0.2239 \\
-0.2258 \\
-0.2270 \\
-0.2274 \\
-0.2269 \\
-0.2255 \\
-0.2232 \\
-0.2198 \\
-0.2155 \\
-0.1794 \\
-0.1244 \\
-0.0614 \\
-0.0020 \\
+0.0455 \\
+0.0774 \\
+0.0672 \\
+0.0506 \\
+0.0394 \\
+0.0315\end{array}$ & $\begin{array}{l}-0.0784 \\
-0.0779 \\
-0.0773 \\
-0.0766 \\
-0.0758 \\
-0.0749 \\
-0.0739 \\
-0.0728 \\
-0.0715 \\
-0.0700 \\
-0.0684 \\
-0.0665 \\
-0.0645 \\
-0.0623 \\
-0.0603 \\
-0.0592 \\
-0.0606 \\
-0.0669 \\
-0.0805 \\
-0.1023 \\
-0.1290 \\
-0.1525 \\
-0.1626 \\
-0.1648 \\
-0.1681 \\
-0.1721 \\
-0.1767 \\
-0.1815 \\
-0.1863 \\
-0.1911 \\
-0.1956 \\
-0.1998 \\
-0.2034 \\
-0.2065 \\
-0.2088 \\
-0.2104 \\
-0.2111 \\
-0.2109 \\
-0.2098 \\
-0.2076 \\
-0.2045 \\
-0.1740 \\
-0.1236 \\
-0.0637 \\
-0.0060 \\
+0.0409 \\
+0.0731 \\
+0.0634 \\
+0.0473 \\
+0.0366 \\
+0.0291\end{array}$ \\
\hline
\end{tabular}

